\title{
Approximation Methods for FO-IMC Controllers for Time Delay Systems
}

\author{
Cristina I. Muresan ${ }^{1}$, Isabela R. Birs ${ }^{1,2,3, *}$, Ovidiu Prodan $^{4}$, Ioan Nascu ${ }^{1}$ and Robin De Keyser ${ }^{2,3}$ \\ ${ }^{1}$ Department of Automation, Technical University of Cluj-Napoca, Cluj-Napoca, Romania \\ ${ }^{2}$ DySC research group on Dynamical Systems and Control Ghent University, Ghent, Belgium \\ ${ }^{3}$ EDT group, member of Flanders Make consortium, Belgium \\ ${ }^{4}$ Department of Civil Engineering, Technical University of Cluj-Napoca, Cluj-Napoca, Romania
}

\begin{abstract}
Fractional Order Internal Model Control (FO-IMC) is among the newest trends in extending fractional calculus to the integer order control. Approximation of the FO-IMC is one of the key problems. Apart from this, when dealing with time delay systems, the time delay needs also to be approximated. All these approximations can alter the closed loop performance of the controller. In this paper, FO-IMC controllers will be tested in terms of the approximation accuracy. The case study is a first order system with time delay. Several scenarios will be considered, aiming for a conclusion regarding the choice of the approximation method as a function of the process characteristics, closed loop performance and FO-IMC fractional order. To approximate the time delay, two extensively used techniques will be considered, such as the series and Pade approximations. These will be compared to a novel approximation technique. An analysis of the test cases presented show that the series approximation proves more suitable in a single scenario, whereas the novel approximation method produces better results for the rest of the test cases.
\end{abstract}

\section{Introduction}

Fractional calculus represents the generalization of the integration and differentiation to an arbitrary real or complex order. Among the first fractional order (FO) control algorithms are the CRONE controller [1] and the FO-PI ${ }^{\lambda} \mathrm{D}^{\mu}$ controller [2]. The popularity of the latter has ever increased with numerous authors demonstrating the capability of such controllers to enhance the robustness and performance of the control systems [3, 4]. Researchers have also incorporated the ideas of fractional calculus in advanced control strategies [5], [6], [7], etc.

The internal model control (IMC) paradigm has also benefitted from combinations with fractional calculus. Different strategies for designing FO-IMC controllers are presented in [8]-[14], where the design is based on inverting a FO model of the process or an integer order model and adding a FO filter. The tuning requires either a bandwidth specifications to be met, disturbance rejection and set-point tracking, etc. Very few experimental results are presented and most tuning rules are defined for single-input-single-output processes. A counter example is [15].

Regardless of the tuning method chosen to design the FO-IMC controller, one major issue is concerned with the actual implementation of the equivalent controller, especially for time-delay systems. For integer order IMC controller, quite frequently the time-delay is approximated using Pade approximations or series approximations. For FO-IMC controllers, the time-delay approximation is complicated by the need to properly approximate also the FO terms. Various discrete-time approximation methods for FO systems exist, either as a direct or indirect approach. A comprehensive review of numerical tools for fractional calculus and FO controls is given in [16]. In [17], an efficient direct approximation method based on the impulse response is presented, where the impulse response is computed based on the frequency response of the FO system. The advantage of the technique is that it can be applied to any type of FO systems to determine its discrete-time approximation.

In this paper, FO-IMC controllers will be tested in terms of the approximation accuracy. The considered case studies are various types of first order plus dead time (FOPDT) systems, the key difference between them consisting in the delay dominance. To approximate the time delay, two extensively used techniques will be considered, such as the series and Pade approximations. These will be compared to the novel approximation technique in [17]. The fractional order part of the FOIMC controller will be approximated in all cases using [17]. There is a single test scenario where the series approximation has higher suitability, whereas the approximation method developed in [17] produces better results for all the other test cases.

The paper is structured as follows. Some brief notion regarding the FO-IMC controllers and their tuning is given in Section 2. Next, the approximation method used in this manuscript will be presented in Section 3.

Corresponding author: Isabela.Birs@aut.utcluj.ro 
Numerical examples are considered in Section 4, while the last section concludes the paper.

\section{Tuning of FO-IMC controllers for FOPDT processes}

A first order time delay system is described by the following transfer function:

$$
H_{P}(s)=\frac{k}{T s+1} e^{s}
$$

The closed loop scheme with the IMC controller is given in Figure 1, where $H_{I M C}(s)$ stands for the FO-IMC controller in (2), $H_{P}(s)$ is the process, $\mathrm{Hm}(\mathrm{s})$ is the process model, $H_{c}(s)$ is the equivalent controller.

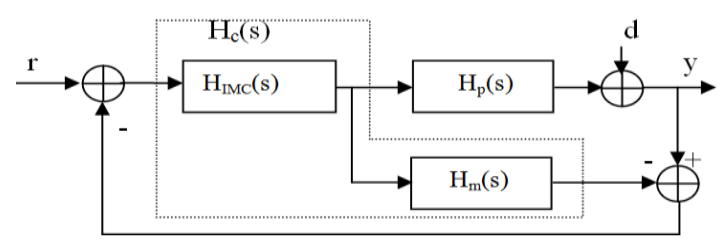

Fig. 1. IMC closed loop control scheme

In a series (first order) approximation of the time delay, with $e^{s} \quad 1 \quad s$, where $\alpha$ is the fractional order, usually in the range $(0 ; 2)$ and $\lambda$ is the IMC filter, the FOIMC and the equivalent controllers are given by:

$$
\begin{gathered}
H_{F O I M C}(s)=\frac{T s+1}{k} \frac{1}{s+1} \\
H_{c}(s)=\frac{H_{F O I M C}(s)}{1 H_{F O I M C}(s) H_{m}(s)}=\frac{T s+1}{k s} \frac{1}{s^{1}+}
\end{gathered}
$$

In a Pade approximation of the time delay, with $e^{s} 1 \frac{1}{2} s / 1+\frac{-1}{2} s$, the FO-IMC and the equivalent controller are determined as:

$$
\begin{array}{r}
H_{F O I M C}(s)=\frac{T s+1}{k} \frac{1+\frac{1}{2} s}{s+1} \\
H_{c}(s)=\frac{(T s+1)\left(1+\frac{-}{2}\right)}{k s} \frac{1}{s^{1}+\frac{1}{2}}
\end{array}
$$

For the novel approximation method in [17], the FOIMC and the equivalent controllers are determined as:

$$
H_{F O I M C}(s)=\frac{T s+1}{k} \frac{1}{s+1}
$$

$$
H_{c}(s)=\frac{T s+1}{k} \frac{1}{\lambda s^{\alpha}+1-e^{-\tau s}}
$$

Notice that in this last case, the time delay has not been approximated and is used as such in the expression of the equivalent controller in (7).

To tune the FO-IMC controller, regardless of the approximation used, the open loop system is firstly computed, $H_{o l}(s)=H_{c}(s) H_{p}(s)$, then, a certain phase margin $P M$ and gain crossover frequency $\omega_{c}$ are imposed in order to determine the two unknown parameters, the fractional order $\alpha$ and the filter time constant $\lambda$ :

$$
\begin{gathered}
\left|H_{o l}\left(\begin{array}{ll}
j_{c} &
\end{array}\right)\right|=1 \\
\angle H_{o l}\left(j \omega_{c}\right)=-\pi+P M
\end{gathered}
$$

In [15], the tuning procedure for an FO-IMC controller, with a series approximation of the time delay, is given. The advantages of using a FO-IMC controller, instead of the classical integer order IMC are highlighted through numerical simulations and an experimental test.

\section{Efficient approximation method for fractional order systems}

The proposed discrete-time approximation method used in this paper has been developed and presented in [17]. The method is suitable for any non-rational transfer function and consists in four steps.

Step 1: Discretize the FO Laplace operator using:

$$
w\left(z^{1}\right)=\frac{1+}{T_{s}} \frac{1 z^{1}}{1+z^{1}}
$$

with $\delta \in[0 \div 1]$ and $T_{s}$-the sampling period. The parameter $\delta$ is a shaping knob and should be selected according to the system to be approximated [17]. This first step produces a discrete time FO system.

Step 2: Calculate the frequency response of the discrete time FO system. To compute the frequency response, the Laplace operator $s$ has to be replaced with $j \omega$, where $=\frac{2}{T_{s} N_{s}}\left[\begin{array}{ccccc}0 & 1 & 2 & \ldots & \frac{N_{s}}{2}\end{array}\right]$. Then, the frequency response of the discrete-time system is computed according to $z^{1}=e^{T_{s} s}$. The parameter $\mathrm{N}_{\mathrm{s}}$ is also a tuning knob. This second step produces a vector of frequency response values of the FO discrete time transfer function.

Step 3: Calculate the impulse response of the discrete time FO system, based on the inverse Fast Fourier Transform (FFT) algorithm. This step results in a vector of $\mathrm{N}_{\mathrm{s}}$ impulse response values:

$$
g[n]=\frac{1}{N_{s}} \sum_{k=0}^{N_{s}-1} G[k] e^{+j \frac{2 \pi}{N_{s} n k}}, n=0,1,2, \ldots, N_{s}-1
$$


Step 4: Determine a rational discrete time transfer function having a similar impulse response as obtained from the inverse FFT. The order $\mathrm{N}$ of the approximation has to be specified. This step results in a rational discrete time transfer function:

$$
G\left(z^{1}\right)=\frac{c_{0}+c_{1} z^{1}+\ldots+c_{N} z^{N}}{d_{0}+d_{1} z^{1}+\ldots+d_{N} z^{N}}
$$

\section{Numerical examples}

The first order time delay system in (1) is considered here, with $k=1$ and $T=1$, in three different scenarios, as indicated in Table 1.

Table 1. Numerical examples

\begin{tabular}{|c|c|c|c|c|}
\hline Example & $\lambda$ & $\tau$ & $\lambda / \tau$ & $\lambda / T$ \\
\hline First & 0.5 & 0.5 & 1 & 0.5 \\
\hline Second & 0.5 & 2 & 0.25 & 0.5 \\
\hline Third & 0.25 & 2 & 0.125 & 0.25 \\
\hline
\end{tabular}

In the first two examples, $\lambda=0.5$, equal to $50 \%$ the original process time constant, $T$, was used, but a different value for the time delay. There is considerable delay dominance for the second process $(\tau / T=2)$, compared to the first one $(\tau / T=0.5)$. A delay dominant process is also chosen in the third example, with $\tau / T=2$, but the filter time constant is now half the one used in examples 1 and 2 , with $\lambda=0.25$ and thus a lower ratio $\lambda / \tau=0.125$. To show the effect of the time delay approximation method, as a function of the fractional order $\alpha$ and ratios $\lambda / \tau$ and $\lambda / T$, we consider the closed loop response to a unit step reference. The simulation results for example 1 are given in Fig. 2-4, for example 2 in Fig. 5-7, while for example 3 in Fig. 8-10.

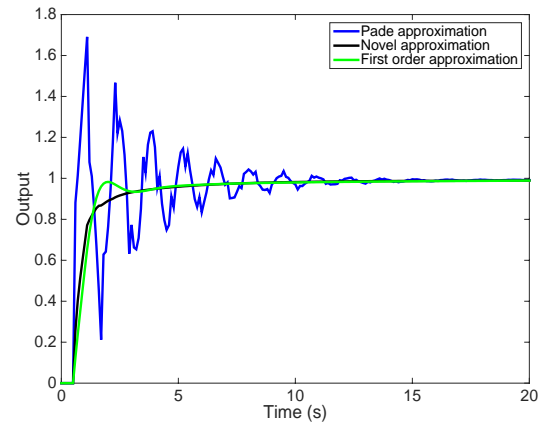

a)

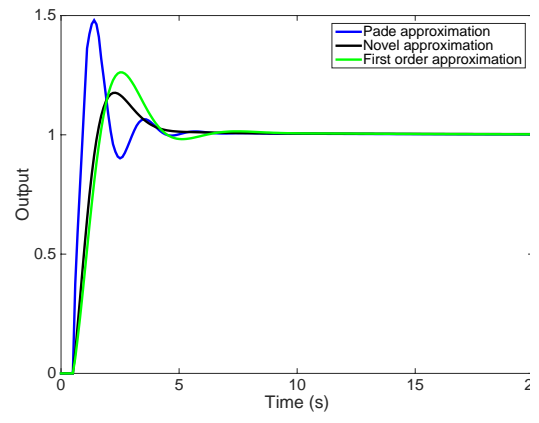

a)
Compare the results for the first and second examples. For the second example, the process exhibits a time delay $\tau=2$, four times larger than in the first example, where $\tau=0.5$. In both examples, the Pade approximation offers the worst results. This is due to the lack of robustness of the Pade approximation, in cases where $\lambda<0.8 \tau$ [18]. Also, notice that the Pade approximation leads to a larger overshoot, a higher settling time, a poorly damped closed loop response and a larger control effort with significant amplitude spikes. In the second example, where $0.8 \tau=1.6$, thus with $\lambda=0.5$ significantly smaller than $0.8 \tau$, the novel approximation method offers the best results, with the smallest overshoot and settling time and the smallest control effort. For the first example, with $\lambda=0.5$, slightly larger than $0.8 \tau=0.4$, both the novel and the first order approximations produce comparable results, although for lower values of the fractional order $\alpha$, the novel approximation method offers better results compared to the first order one. Notice also the tendency of the Pade approximation to go unstable for fractional orders with larger deviations from $\alpha=1$.

In the third example, the filter time constant has been chosen even smaller compared to the second example. In this case, the simulation results in Fig. 8-10 show that the novel approximation method is the best choice. The first order approximation can be considered as a suitable alternative for fractional orders $\alpha>1.8$.

In all cases, the novel approximation method has been considered for the approximation of the fractional order terms in the FO-IMC controller and the same order of magnitude for the controllers has been selected, $N=5$. The sampling period $T_{s}=0.1$ seconds has been considered in the discrete-time approximation of the equivalent controllers. Notice that in all cases presented, $\lambda$ has been chosen smaller than the corresponding process time constant. This is due to the fact that a larger value for $\lambda$ will result in a small closed loop bandwidth. Hence, the approximation error of the process time delay at high frequencies becomes of less importance because it occurs at frequencies, which are out of the passband. 


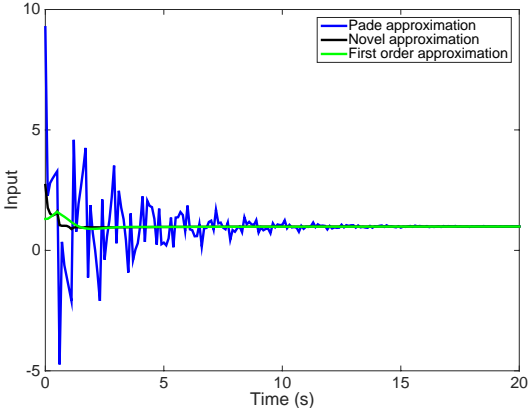

b)

Fig. 2. First example step response with $\alpha=0.8$ a) output and b) input signals

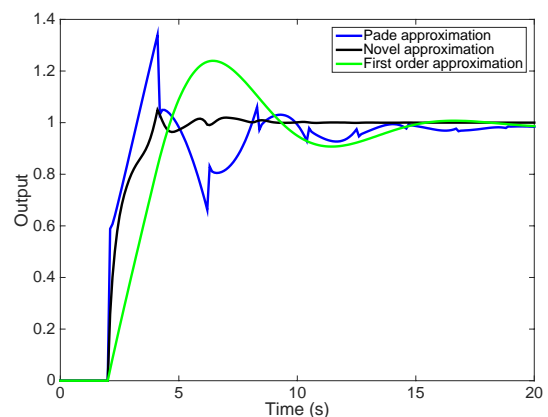

a)

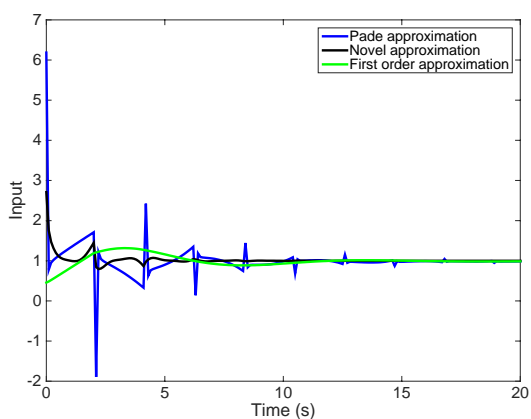

b)

Fig. 5. Second example step response with $\alpha=0.8$ a) output and b) input signals

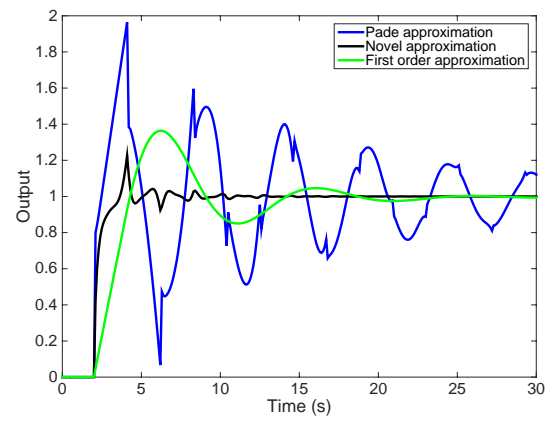

a)

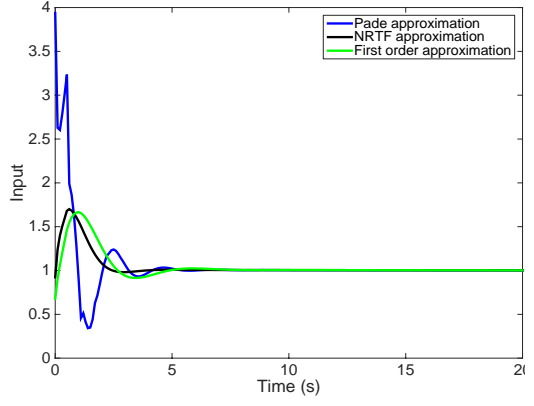

b)

Fig. 3. First example step response with $\alpha=1.3$ a) output and $b$ ) input signals

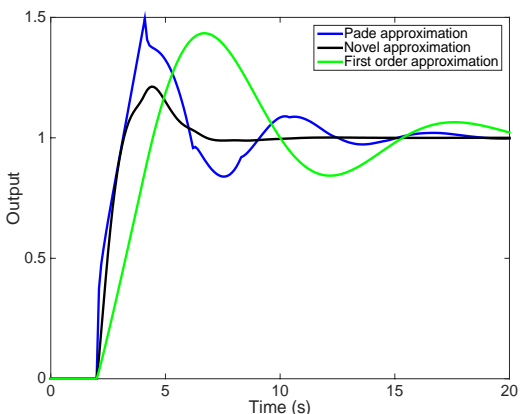

a)

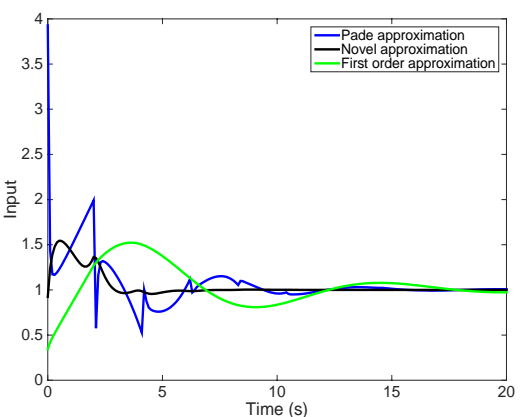

b)

Fig. 6. Second example step response with $\alpha=1.3$ a) output and $b$ ) input signals

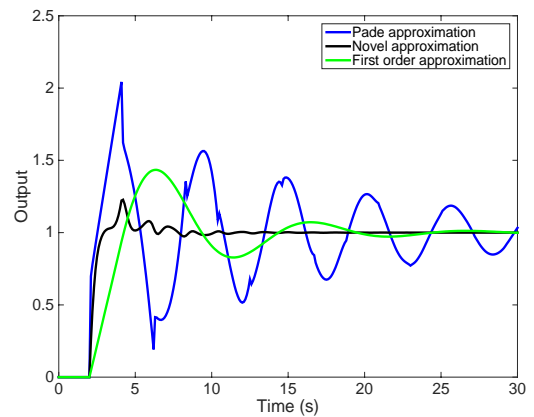

a)

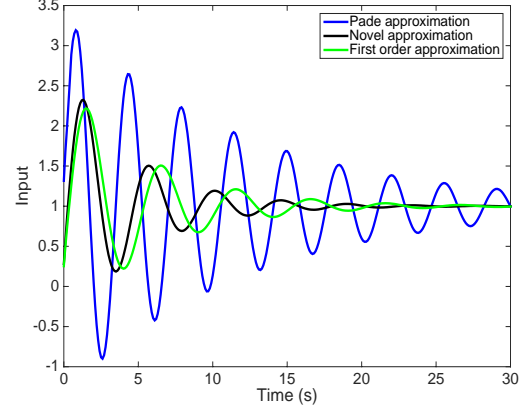

b)

Fig. 4. First example step response with $\alpha=1.8$ a) output and b) input signals

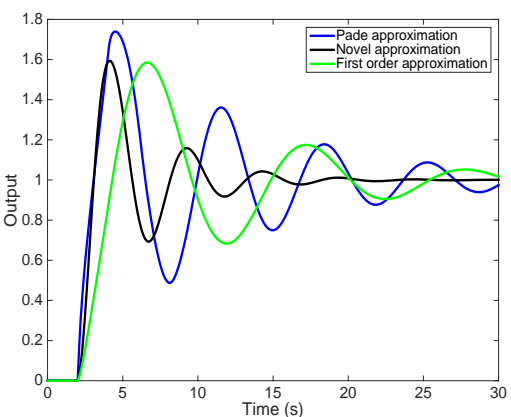

a)

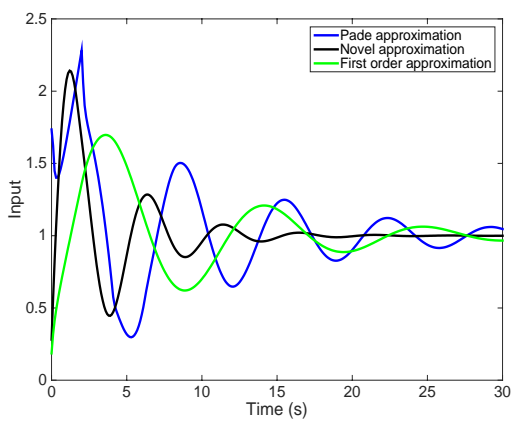

b)

Fig. 7. Second example step response with $\alpha=1.8$ a) output and b) input signals

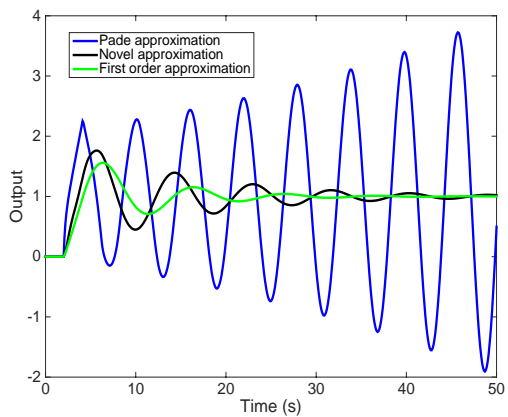

a) 


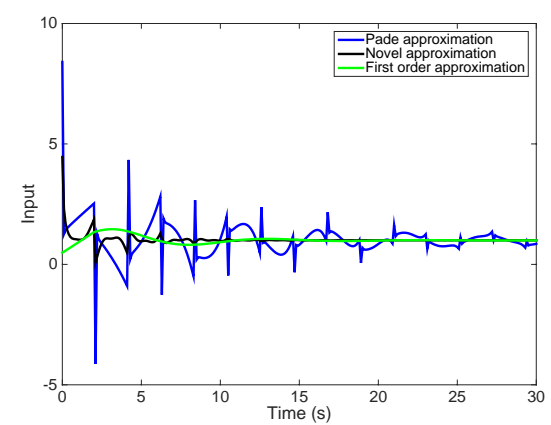

b)

Fig. 8. Third example step response with $\alpha=0.8$ a) output and b) input signals

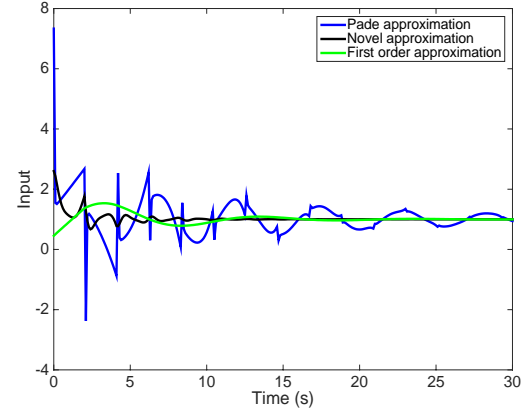

b)

Fig. 9. Third example step response with $\alpha=1.1 \mathrm{a}$ ) output and b) input signals

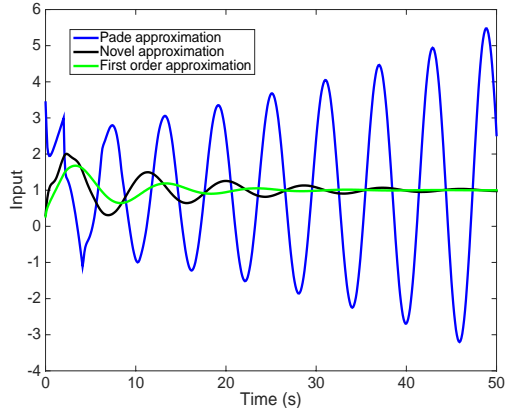

b)

Fig. 10. Third example step response with $\alpha=1.8$ a) output and b) input signals
Three numerical examples have been considered in this paper. The second and third examples are both delay dominant processes, with the ratio $\tau / T=2$ and $\tau / t_{s}=0.34$, where $t_{s}$ is the process settling time. In this case, for processes with significant time delays, the novel approximation method is a better option compared to the first or the Pade approximation methods. For processes such as the first example (lag dominant), with $\tau / T=0.5$ and $\tau / t_{s}=0.11$, the first order and the novel approximation methods can be used for similar results.

Extending the simulation results presented here, for processes with an even more prominent lag dominance (ratio $\tau / T>0.5$, as used in the first example), the novel and first order approximation methods will produce even more similar results, regardless of the choice of the fractional order $\alpha$ and the FO-IMC filter time constant $\lambda$. For processes with a more prominent delay dominance (ratio $\tau / T>2$, as used in the second and third examples), the novel approximation method will provide even better closed loop results than the first order approximation. It is also possible to improve the closed loop results by considering a higher order of the approximation. The Pade approximation method offers uninteresting results in approximating the time delay in a FO-IMC control loop, when used on processes with $\lambda<0.8 \tau$, as it has also been concluded in the integer order case [18].

\section{Conclusions}

The FO-IMC controller has the advantage of increasing the robustness of the traditional IMC due to the supplementary tuning parameter involved, the fractional order. The key problem with FO-IMC controllers is represented by the approximation of the FO terms. Apart from this, the time delay needs also to be approximated. All these approximations can alter the closed loop performance of the controller. In this paper, FO-IMC controllers have been tested in terms of the approximation accuracy. The case study is a first order system with time delay. Scenarios such as lag or time delay dominance have been included. To approximate the FO terms in the FO-IMC controller, a novel approximation method has been used. Apart from these, the process time delay has been approximated using the series and Pade approximations. The only difference between the three FO-IMC implementation methods is the approximation of the process time delay. Hence, for a noticeable difference between the three approximation methods, a significant time delay was considered. The Pade approximation method led to poor closed loop response. The series and novel approximation methods led to similar results in the case of lag dominant processes, whereas for delay dominant ones, the novel method proved to be superior to the series approach.

\section{Acknowledgement}

This research has been financed by a grant of the Romanian National Authority for Scientific Research and Innovation, CNCS/CCCDI-UEFISCDI, project number PN-III-P1-1.1-TE2016-1396, TE 65/2018.

\section{References}

1. Oustaloup, A., Sabatier, J., \& Lanusse, P. (1999). From fractional robustness to CRONE control. Fractional Calculus and Applied Analysis, 2, 1-30

2. Podlubny, I. (1999). Fractional-order systems and $\mathrm{PI} \lambda \mathrm{D} \mu$ controllers. IEEE Transactions on Automatic Control, 44, 208-214

3. Monje, C.A., Chen, Y.Q., Vinagre, B.M., Xue, D., \& Feliu, V. (2010). Fractional order Systems and Controls: Fundamentals and Applications, London: Springer-Verlag

4. Muresan, C.I., Ionescu, C., Folea, S., De Keyser, R. (2015). Fractional Order Control of Unstable Processes: The Magnetic Levitation Study Case, Nonlinear Dynamics, Vol. 80, No. 4, 1761-1772

5. Ding, Y., Wang, Z., \& Ye, H. (2012). Optimal Control of a Fractional-Order HIV-Immune System With Memory, IEEE Transactions on Control Systems Technology, 20, 763-769

6. Luo, Y., Chen, Y.Q., Ahn, H., \& Pi, Y. (2012). Fractional order periodic adaptive learning compensation for the state-dependent periodic disturbance, IEEE Transactions on Control Systems Technology, 20, 465-472

7. De Keyser, R., Muresan, C.I., Ionescu, C. (2016), A Novel Auto-tuning Method for Fractional Order PI/PD Controllers, ISA Trans., Vol. 62, 268-275 
8. Vinopraba, T., Sivakumaran, N., \& Narayanan, S. (2011). IMC Based Fractional order PID Controller, IEEE International Conference on Industrial Technology (ICIT)

9. Tavakoli-Kakhki, M., \& Haeri, M. (2011). Fractional order model reduction approach based on retention of the dominant dynamics: Application in IMC based tuning of FOPI and FOPID controllers. ISA Trans, $50,432-442$

10. Maâmar, B., \& Rachid, M. (2014). IMC-PIDfractional-order-filter controllers design for integer order systems. ISA Trans, 53, 1620-1628

11. Valerio, D., \& Sa da Costa, J. (2006). Tuning of fractional PID controllers with Ziegler-Nichols-type rules, Signal Processing, 86, 2771-2784

12. Vinopraba, T., Sivakumaran, N., Narayanan, S., \& Radhakrishnan, T.K. (2012). Design of internal model control based fractional order PID controller. J Control Theory Appl., 10, 297-302

13. Isfer, L.A.D., da Silva, G.S., Lenzi, M. K. \& Lenzi, E.K. (2012). Generalization of internal model control loops using fractional calculus, Latin American Applied Research, 42

14. Sondhi, S., \& Hote, Y.V. (2014). Fractional IMC design for fractional order gas turbine model, The 9th International Conference on Industrial and Information Systems (ICIIS), Dec. 15-17 2014

15. Muresan, C.I., Dutta, A., Dulf, E.H., Pinar, Z., Maxim, A., Ionescu, C.M. (2016), Tuning algorithms for fractional order internal model controllers for time delay processes, International Journal of Control, Vol. 89, No. 3, pp. 579-593

16. Li Z, Liu L, Dehghan S, et al. (2017), A review and evaluation of numerical tools for fractional calculus and fractional order controls. Int J Contr; 90(6): 1165-81.

17. De Keyser, R., Muresan, C.I., Ionescu, C.M. (2018), An efficient algorithm for low-order discrete-time implementation of fractional order transfer functions, ISA Transactions, vol. 74, pp. 229-238

18. Rivera, D.E., M. Morari and S. Skogestad (1986). Internal Model Control. 4. PID Controller Design, Ind. Eng. Chem. Proc. Des. Dev., 25, 252-265 